\title{
CRITICAL SCALE FOR A CONTINUOUS AIMD MODEL
}

\author{
ILIE GRIGORESCU AND MIN KANG
}

\begin{abstract}
A scaled version of the general AIMD model of transmission control protocol (TCP) used in internet traffic congestion management leads to a Markov process $x(t)$ representing the time dependent data flow that moves forward with constant speed on the positive axis and jumps backwards to $\gamma x(t), 0<\gamma<1$ according to a Poisson clock whose rate $\alpha(x)$ depends on the interval swept in between jumps. We give sharp conditions for Harris recurrence and analyze the convergence to equilibrium on multiple scales (polynomial, fractional exponential, exponential) identifying the critical case $x \alpha(x) \sim \beta$. Criticality has different behavor according to whether it occurs at the origin or infinity. In each case we determine the transient (possibly explosive), null- and positive - recurrent regimes by comparing $\beta$ to $(-\ln \gamma)^{-1}$.
\end{abstract}

\section{Introduction}

Let $(\Omega, \Sigma, P)$ be a probability space and $\{x(t)\}_{t \geq 0}$ a stochastic process adapted to the filtration $\left\{\mathcal{F}_{t}\right\}_{t \geq 0}$ on $\Sigma$. In the following we study the time-homogeneous one particle process $\{x(t)\}_{t \geq 0}$ with state space $(0, \infty)$ solving the martingale problem with generator $(B, \mathcal{D})$

$$
B \phi(x)=\phi^{\prime}(x)+\alpha(x)(\phi(\gamma x)-\phi(x)), \quad \phi \in \mathcal{D}=C_{b}^{1}((0, \infty))
$$

where $\gamma \in(0,1), C_{b}^{k}((0, \infty))$ is the space of functions with $k$ continuous derivatives up to the boundary of $(0, \infty)$, and $\alpha(x)$ is a measurable, nonegative function.

This simple dynamics is the scaled version $[9,15,11,6]$ of an additive increase multiplicative decrease (AIMD) process modeling the traffic flow in internet congestion control

Date: September 26, 2012.

1991 Mathematics Subject Classification. Primary: 60K30; Secondary: 60J25, 90B20.

Key words and phrases. AIMD, TCP, Harris recurrence, geometric ergodicity, local Doeblin condition, criticality. 
$[2,7,8,1,11,3]$. For a recent approach, a primer on the model and biography we mention [3]. Besides its original formulation introduced in [2], the model has applications in questions of distributed resource allocation [16].

Even though we have been introduced to the subject by $[7,8]$ our approach lies towards the pure spectrum of applied mathematics, with emphasis on the critical threshold for recurrence and the analysis of the speed of convergence to equilibrium in sub-exponential regimes.

Let $\tau_{i}^{\prime}, i=0,1, \ldots$ be a non-decreasing sequence of random times representing the loss events (when packets of data are lost). Between two consecutive loss events (jumps) $\tau_{i-1}^{\prime} \leq$ $t<\tau_{i}^{\prime}$, the transmission rate (also known as congestion window or cwnd) $x(t)$ increases at constant speed one. Once the volume $\int_{\tau_{i-1}^{\prime}}^{t} \alpha(x(s)) d s$ reaches a random quantity $\chi_{i}>0$ as $t \uparrow \tau_{i}^{\prime}$, the rate falls back to $\gamma x\left(\tau_{i}^{\prime}-\right)$. This mechanism leads to the construction of a Markov process based on a sequence of i.i.d. exponential r.v. $\chi_{1}, \chi_{2}, \ldots$ corresponding to the inter-arrival times of a Poisson process with intensity one. Starting at $x(0)=x>0$, let $w_{1}^{\prime}, w_{2}^{\prime}, \ldots$ be the actual holding times of the process and $\tau_{0}^{\prime}=0, \tau_{1}^{\prime}, \tau_{2}^{\prime}, \ldots$ be the actual jump times of $x(t)$. More precisely, take $w_{0}^{\prime}=0$ and for $j \geq 1$,

$$
\tau_{j}^{\prime}=\inf \left\{t>\tau_{j-1}^{\prime} \mid \chi_{j}<\int_{\tau_{j-1}^{\prime}}^{t} \alpha(x(s)) d s\right\}, \quad w_{j}^{\prime}=\tau_{j}^{\prime}-\tau_{j-1}^{\prime},
$$

with the infimum over the empty set equal to $+\infty$. Let's denote $x_{0}=x$ and $x_{j}=x\left(\tau_{j}^{\prime}\right)$ the position of the process right after the $j$-th jump. In the interval $s \in\left[\tau_{j-1}^{\prime}, \tau_{j}^{\prime}\right), x(s)=$ $x_{j-1}+\left(s-\tau_{j-1}^{\prime}\right)$, which gives, for $j \geq 1$,

$$
x_{j}=\gamma\left(x_{j-1}+w_{j}^{\prime}\right), \quad \int_{x_{j-1}}^{x_{j-1}+w_{j}^{\prime}} \alpha(z) d z=\chi_{j}
$$

and

$$
x_{j}=\gamma^{j} x+\sum_{k=0}^{j-1} \gamma^{k+1} w_{j-k}^{\prime} .
$$

Naturally, in applications, the problem is interesting when $\alpha(x)>0$ for sufficiently large $x$. On the patches where $\alpha(x)$ vanishes, the particle moves deterministically at rate one, eventually reaching the support of $\alpha(x)$. Without loss of generality we shall assume that $\alpha(x)$ does not vanish in a neighborhood of the origin. When $\alpha(x)=1-\mathbf{1}_{[\gamma a, a]}(x)$, any state $x<\gamma a$ is transient. Additionally, if the integral is infinite over over a bounded interval, no larger values are ever reached. 
To prevent such behavior, the rate function $\alpha:(0, \infty) \rightarrow \mathbb{R}$ will be assumed nonnegative, locally integrable and bounded away from zero on any compact set. For any $0<a<b$

$$
\text { (i) } \quad \alpha_{0}([a, b]):=\inf _{x \in[a, b]} \alpha(x)>0,
$$

(ii) $\int_{a}^{b} \alpha(x) d x<\infty$.

These bounds are essentially irreducibility conditions.

Under (1.5)(ii), we denote $A(x)=\int_{1}^{x} \alpha(z) d z$, where the point $z=1$ is chosen for convenience only and we note that $A(x)$ is continuous and increasing.

Integrability is captured by one of the limits

$$
\text { (i) } \lim _{x \rightarrow 0^{+}} A(x)>-\infty, \quad \text { (ii) } \lim _{x \rightarrow \infty} A(x)=+\infty .
$$

We shall say that $\alpha(x)$ satisfies the growth condition at zero if if there exists $b_{0}>0$ such that

$$
\mu_{+}=\sup _{x \leq b_{0}}\{A(x)-A(\gamma x)\}<1 .
$$

and that $\alpha(x)$ satisfies the growth condition at infinity if there exists $a_{0}>0$ such that

$$
\mu_{-}=\inf _{x \geq a_{0}}\{A(x)-A(\gamma x)\}>1 .
$$

Since the function $A(x)$ is nondecreasing, we have the implications

$$
\text { (1.6) (i) } \Rightarrow(1.7) \text { and (1.8) } \Rightarrow(1.6) \text { (ii) . }
$$

Theorem 1 (the most general result) requires the milder conditions (1.7) and (1.6) (ii), while Theorem 2 (main result) requires the more stringent (1.6) (i) and (1.8).

As expected, the conditions at zero say that the rate $\alpha(x)$ cannot be too large when $x$ is small, otherwise the process would undergo many jumps backwards in the neighborhood of zero, and drift to this endpoint, or even have infinitely many jumps in finite time, called explosion (the transition functions are defective). At $+\infty$, the conditions require that $\alpha(x)$ be not too small, otherwise allowing the process to wander away to infinity, or have only a finite number of jumps.

Examples of locally integrable functions satisfying (1.5 (i)) - (1.8) are given by either of the following:

(i) $\alpha(x)$ nondecreasing, finite and strictly positive on $(0, \infty)$;

(ii) $\alpha(x)$ continuous, positive and bounded away from zero at infinity;

(iii) $\alpha(x) \sim x^{p}$ at both zero and infinity, when $p>-1$; 
(iv) $\alpha(x)$ integrable at zero and $\alpha(x) \sim \beta x^{-1}, \beta>0$ as $x \rightarrow \infty$, with $\beta>\beta^{*}$, where $\beta^{*}$ is a critical value depending on $\alpha(x)$ separating two recurrence regimes within the critical case with exponent $p^{*}=-1$ (see Theorem 2).

It is to be noted that $\alpha(0+)$ may be infinite $(-1<p<0)$, constant $(p=0)$, and zero $(p>0)$.

Explicit calculations for power law function $\alpha(x)$ with $p \geq 0$ are done in [1]. Due to homogeneity, the transformation $y(t)=A(x(t))$ brings down the invariant measure to the case $\alpha=$ constant. Even though [3] studies a nonlinear (AINMD) model, in which the back-off function (giving the multiplicative decrease position after jump) $\gamma(x) \neq \gamma \cdot x$ is non-linear, the present discussion can be largely extrapolated because, in applications, $\gamma(x)$ is assumed Lipschitz continuous.

The growth rate (1.8) appears naturally as a Lyapunov - type condition for recurrence. It is easy to see that when $\alpha(x)$ is continuous, (1.8) guarantees that $A(x(t))$, stopped as soon as it reaches a value below $a_{0}$, is a super-martingale. The example $\alpha(x)=\beta x^{-1}$ as $x \rightarrow \infty$ from Theorem 4 shows that it is also a sharp recurrence condition, in the sense that for $\beta<(-\ln \gamma)^{-1}$ the process is transient.

The divergence/convergence of the integral at infinity also gives a sharp phase transition, this time for having an infinite/finite number of jumps. As soon as $\alpha(x) \sim x^{p}, p<-1$, for $x \rightarrow \infty$, with positive probability, the process will undergo only a finite number of jumps, and will drift to infinity at speed one after that.

Under stronger conditions like $0<\alpha_{0} \leq \alpha(x) \leq\|\alpha\|<\infty$, all main results on exponential ergodicity, including the existence of a bounded density for the invariant measure, can be obtained [10] by coupling with a process with constant rate $\alpha$.

We found useful to provide a brief summary of the local Doeblin theory for continuous time Markov processes on general state spaces in Section 6, based mainly on [4], [12] and, to a lesser extent on $[5,13,14]$.

We are ready to introduce the main results of the paper. The Lebesgue measure on $(0, \infty)$ is denoted by $l(d x)$ and recurrence means $l(d x)$ - Harris recurrence in the sense of $[14,12]$.

Theorem 1. Assume $\alpha(x)$ satisfies (1.5) (ii). With probability one (i) if $\alpha(x)$ satisfies (1.6) (ii), then there are infinitely many jumps and 
(ii) if, in addition, $\alpha(x)$ satisfies (1.7), then $\lim _{n \rightarrow \infty} \tau_{n}^{\prime}=+\infty$, i.e. the process is nonexplosive.

Proof. The proof is contained in Subsection 2.1.

Theorem 2. Assume (1.5), (1.7) and (1.8) are satisfied. Then, the process with generator (1.1) is $l(d x)$ - Harris recurrent. If we replace (1.7) with the stronger condition (1.6)(i), then the process satisfies the local Doeblin condition for an attractive small set that may be chosen equal to a closed interval. In addition, (ii) there exists $\beta^{*}>0$ such that if $x \alpha(x) \geq \beta^{*}$ for all sufficiently large $x$, then the process is positive recurrent, and (iii) if $\alpha(x)$ is bounded away from zero as $x \rightarrow \infty$, then the process is exponentially ergodic.

Remark. We note that $(-\ln \gamma)^{-1} \leq \beta^{*} \leq 2(1-\gamma)^{-1}$, where $\beta^{*}$ is defined in $(5.7)$.

The next theorem analyzes the case of $\alpha(x)$ on an intermediate scale. We note that the power law with $p \geq 0$ is covered by Theorem 2 (iii).

Theorem 3. Assume (1.5), (1.6)(i) and that there exists $\beta>0, a_{1}>0$ and $p \in(-1,0)$ such that $\alpha(x) \geq \beta x^{p}$ for all $x \geq a_{1}$. Then, Theorem 2 (i) is satisfied and for sufficiently large $a^{\prime}$, the fractional exponential moments of $\tau_{\left(0, a^{\prime}\right]}$ are uniformly bounded, i.e. (5.3) is true for $B(t)=\exp \left(t^{r}\right), r \in[0,1+p)$, and if $\beta$ is sufficiently large, the moments extend to the value $1+p$. The power law is sharp in the sense that when $\alpha(x)=x^{p}, p \in(-1,0)$ as $x \rightarrow \infty, \tau_{\left(0, a^{\prime}\right]}$ has no finite exponential moment for any $a^{\prime}>0$.

Remark. It is immediate that in this case, convergence to the invariant measure is stronger than polynomial of any order, but not exponential.

Proof. The proof is in Subsection 5.2.

As suggested by Theorem 2 (ii), the power law with exponent $p^{*}=-1$ is critical. On this scale, the critical value is not the exponent, but $\beta=(-\ln \gamma)^{-1}$, giving rise to two layers of criticality. Theorem 4 makes this precise: The rate function $\alpha(x)=\beta x^{-1}$ generates a process that can be transient, recurrent or positively recurrent for different ranges of $\beta>0$.

We say that $\alpha(x)$ is critical at infinity (at zero) if there exist $\beta>0, a_{1}>0$ such that $\alpha(x)=\beta x^{-1}$ for all $x \geq a_{1}\left(x \leq a_{1}\right)$. We notice that condition (1.8) when $\alpha(x)$ is critical at infinity is equivalent to $\beta>(-\ln \gamma)^{-1}$, while (1.7) when $\alpha(x)$ is critical at zero is equivalent to $\beta<(-\ln \gamma)^{-1}$. 
Theorem 4. a) Assume $\alpha(x)$ is critical at infinity and (1.5), (1.6) (i) are satisfied. Then

(i) the process $(x(t))$ is transient if $\beta<(-\ln \gamma)^{-1}$, recurrent if $\beta \geq(-\ln \gamma)^{-1}$, and $x>a^{\prime}$, then

(ii) if $\beta>(1-\gamma)^{-1}$, then $E_{x}\left[\tau_{\left(0, a^{\prime}\right]}\right]<\infty$ (positive recurrent).

(iii) if $\beta \leq(1-\gamma)^{-1}$, then $E_{x}\left[\tau_{\left(0, a^{\prime}\right]}\right]=+\infty$.

b) Assume $\alpha(x)$ is critical at zero and the growth condition at infinity (1.8) is satisfied. Then the process $(x(t))$ is transient if $\beta>(-\ln \gamma)^{-1}$ and recurrent if $\beta \leq(-\ln \gamma)^{-1}$.

Remarks. 1) An exact formula for $E_{x}\left[\tau_{\left(0, a^{\prime}\right]}\right]$ is given in (5.12). 2) In case a) it is relevant that $(-\ln \gamma)^{-1}<(1-\gamma)^{-1}$. Evidently in case b) (1.6) (i) is not satisfied. This gives a nontrivial example when $\alpha(x)$ is not integrable at zero yet the process is non-explosive, which is relevant for Theorem 1 (ii).

The exact critical case at both ends is the only theorem we prove in this section.

Theorem 5. If $\alpha(x)=\beta x^{-1}, \beta>0$, for all $x>0$, then the process is non-explosive and transient if $\beta<(-\ln \gamma)^{-1}$, non-explosive and null recurrent if $\beta=(-\ln \gamma)^{-1}$, and explosive converging in finite time to zero when $\beta>(-\ln \gamma)^{-1}$.

Proof. When $\alpha(x)=\beta x^{-1}$, the growth constants from (1.8),(1.7) are the same, $\mu_{-}=$ $-\beta \ln \gamma=\mu_{+}$. The positions $\left(x_{n}\right)$ right after jump $n$ can be mapped into a random walk $\ln \left(x_{n}\right), n \geq 0$ on the real line. One can see that from (1.3), $\ln \left(\gamma^{-1} x_{n}\right)-\ln \left(x_{n-1}\right)=$ $\beta^{-1} \chi_{n}$, where $\left(\chi_{n}\right)_{n \geq 1}$ are i.i.d. mean one exponential times. It follows that $x_{n}=$ $x_{0} \gamma^{n} \exp \left(\beta^{-1} \sum_{j=1}^{n} \chi_{j}\right)$, the exponential of a random walk with increments $\beta^{-1} \chi+\ln \gamma$, having a drift equal to $\beta^{-1}\left(1-\mu_{-}\right)$, with critical value $\mu_{-1}=1$, or equivalently, $\beta=(-\ln \gamma)^{-1}$.

The actual holding time between jumps $w_{n}^{\prime}=\gamma^{-1} x_{n}-x_{n-1}$ when starting at $x_{n-1}$, is equal to $x_{n-1}\left(\exp \left(\chi_{n} / \beta\right)-1\right)$. We note that for all $\beta \in(0,1]$ this time has infinite expected value.

Even though the random walk always has infinitely many steps (corresponding to jumps), the process $x(t)$ might be explosive when infinitely many jumps occur in finite time, i.e. the transition kernel does not integrate to one for all times $t>0$. Explosion coincides with the event that $\sum_{j \geq 1} w_{j}^{\prime}<\infty$, which is equivalent to $\sum_{j \geq 1} x_{j}<\infty$.

Using the root test with the formula of $x_{n}$, via the law of large numbers, we can see that in the supercritical case $\beta>(-\ln \gamma)^{-1}$ the process is explosive (infinitely many jumps 
towards zero in finite time) and in the subcritical case $\beta<(-\ln \gamma)^{-1}$ the process is not explosive but drifts to $+\infty$ almost surely.

In the critical case the process is non-explosive, since the series diverges a.s. (based on the root test together with the law of the iterated logarithm) and the chain at $\left(x_{n}\right)$ is null-recurrent. The process is also $l(d x)$ - Harris recurrent based on Propositions 1 and 2 .

\section{Regularity properties}

2.1. Proof of Theorem 1. We shall show that for any $n$, the probability that there are exactly $n$ jumps is zero. This is equal to

$$
P_{x}\left(\tau_{n+1}^{\prime}=+\infty\right)=P_{x}\left(w_{n+1}^{\prime}=+\infty\right)=\int_{0}^{\infty} P_{x}\left(w_{n+1}^{\prime}=+\infty \mid x\left(\tau_{n}^{\prime}\right)=x^{\prime}\right) P\left(x\left(\tau_{n}^{\prime}\right) \in d x^{\prime}\right)
$$

but the integrand is dominated by $P_{x^{\prime}}\left(\chi_{n+1}=+\infty\right)=0$ since $A(+\infty)=+\infty$, for any possible $x^{\prime}>0$, which proves (i).

To prove part (ii), we make the observation that $x(t) \leq t+x$ pathwise. With this in mind, if there were infinitely many jumps before time $T>0$, then we shall show that $\lim _{n \rightarrow \infty} x_{n}=0$. Let $c>T(1-\gamma)^{-1}$ be a large but fixed number. We shall show the statement for a starting point $x \leq c$. Since $c$ is arbitrary, this will prove (ii).

Suppose $\lim _{\sup } \rightarrow \infty x_{n}>0$.

Denote by $B_{m}$ the event that the sequence $x_{n}$ would have infinitely many points greater or equal to $\epsilon=1 / m, m \geq 1$, intersected with the event that there are infinitely many jumps before time $T$. We want to show that $P_{x}\left(B_{m}\right)=0$ for all $m$, which would imply the desired limit equals zero almost surely. Without loss of generality we may assume $x \geq 1 / m$ for the starting point $x$. Since $m$ is fixed in the proof, to simplify notation, let $B_{m}=B$. On $B$, the process returns to the set $\left[\frac{1}{m}, \infty\right)$ infinitely many times before $T$. Let $\tau^{+}$be the first such time coming after the first jump $\tau_{1}^{\prime}$, i.e. $\tau^{+}=\inf \left\{t>\tau_{1}^{\prime} \mid x(t) \geq \frac{1}{m}\right\}$. By construction, on $B, \tau^{+} \leq T$. Then $B \subseteq \theta_{\tau^{+}} B \cap\left\{\tau^{+} \leq T\right\}$, where $\theta$ is the shift operator on the path space.

By construction, $x\left(\tau^{+}\right)$either equals $1 / m$ if $x\left(\tau_{1}^{\prime}\right)<1 / m \leq c$ or equals $x_{1}=\gamma(x+$ $\left.\tau_{1}^{\prime}\right) \leq \gamma(c+T) \leq c$ if $x\left(\tau_{1}^{\prime}\right) \geq 1 / m$, where we used that $c>T /(1-\gamma)$. In both cases $x\left(\tau^{+}\right) \in\left[m^{-1}, c\right]$.

Applying the strong Markov property we derive the upper bound

$$
P_{x}(B)=E_{x}\left[\mathbf{1}_{B}\right] \leq E_{x}\left[E_{x}\left[\theta_{\tau^{+}} \mathbf{1}_{B} \cdot \mathbf{1}_{[0, T]}\left(\tau^{+}\right) \mid \mathcal{F}_{\tau^{+}}\right]\right]
$$




$$
\leq E_{x}\left[E_{x\left(\tau^{+}\right)}\left[\mathbf{1}_{B}\right] \mathbf{1}_{[0, T]}\left(\tau^{+}\right)\right] \leq \sup _{x^{\prime} \in\left[m^{-1}, c\right]} P_{x^{\prime}}(B) P_{x}\left(\tau^{+} \leq T\right) .
$$

Notice that $\tau_{1}^{\prime} \leq \tau^{+}$, and thus the last factor in the inequality has upper bound $P_{x}\left(\tau_{1}^{\prime} \leq T\right)$. Since $\tau_{1}^{\prime}=w_{1}^{\prime}$ and on $[0, T]$ the process cannot exceed $x+T$ by construction, we have

$$
P_{x}\left(\tau_{1}^{\prime} \leq T\right) \leq 1-e^{-(A(x+T)-A(x))} \leq 1-e^{-\left(A(c+T)-A\left(\frac{1}{m}\right)\right)}=p^{\prime}<1 .
$$

Taking the supremum on $x \in\left[m^{-1}, c\right]$ on the left hand side, we obtain $\sup _{x \in\left[m^{-1}, c\right]} P_{x}(B)=$ 0 , which concludes the first step of the proof.

It remains to show that the event $\left\{\lim _{n \rightarrow \infty} x_{n}=0\right\}$ has probability zero. We shall prove the stronger statement that $\left\{\lim \sup _{n \rightarrow \infty} x_{n} \leq \gamma b_{0}\right\}$ has probability zero.

On this event, there exists a rank $n^{\prime}$ depending on $\omega$ such that after $\tau_{n^{\prime}}^{\prime}$, the process never exceeds $b_{0}$, the threshold from (1.7). For $n \geq n^{\prime}$ we have the inequality $A\left(\gamma^{-1} x_{n}\right)-$ $A\left(x_{n-1}\right)=A\left(\gamma^{-1} x_{n}\right)-A\left(x_{n}\right)+A\left(x_{n}\right)-A\left(x_{n-1}\right) \leq \mu_{+}+A\left(x_{n}\right)-A\left(x_{n-1}\right)$.

From $A\left(\gamma^{-1} x_{n}\right)-A\left(x_{n-1}\right)=\chi_{n}$, we see that

$$
\sum_{i=n^{\prime}}^{n} \chi_{i} \leq n \mu_{+}+A\left(x_{n}\right)-A\left(x_{n^{\prime}}\right) \leq\left(n-n^{\prime}\right) \mu_{+}+A\left(\gamma b_{0}\right)-A\left(x_{n^{\prime}}\right) \quad \text { a.s. }
$$

for sufficiently large $n$. It follows that

$$
\left\{\limsup _{n \rightarrow \infty} x_{n} \leq \gamma b_{0}\right\} \subseteq\left\{\limsup _{n \rightarrow \infty} \frac{\sum_{j=1}^{n} \chi_{j}}{n} \leq \mu_{+}\right\},
$$

the last event being negligible by the law of large numbers.

\section{Recurrence}

The next results regard the recurrence of the process. In our case, Section 6 applies with $\mathcal{S}=(0, \infty)$ and $\phi(d x)$ the Lebesgue measure $l(d x)$ on $(0, \infty)$. In the proof of Theorem 2 (subsection 5.1) we show that $l(d x)$ is the maximal irreducibility measure $\psi$.

Proposition 1. Assume conditions (1.5) and (1.8). For any $c>0$ and any $x \in(0, \infty)$, $P_{x}\left(\tau_{(0, c)}<\infty\right)=P_{x}\left(\tau_{(0, c]}<\infty\right)=1$. If $c \geq \gamma^{-1} a_{0}$, where $a_{0}$ is defined in (1.8), then the number of jumps after which the process reaches $(0, c)$ has an exponential moment. Moreover, for any open set $A$ in $(0, \infty)$, we have $P_{x}\left(\tau_{A}<\infty\right)>0$.

Proof. Step 1. We first prove the proposition for $c>\gamma^{-1} a_{0}$. To simplify notation, we put $a^{\prime}=\gamma^{-1} a_{0}$ and $\tau_{0}=\tau_{\left(0, a^{\prime}\right)}$. Pick an arbitrary $\epsilon>0$. 
Relation (1.3) implies

$$
\chi_{j}=A\left(\gamma^{-1} x_{j}\right)-A\left(x_{j-1}\right) \geq \mu_{-}+A\left(x_{j}\right)-A\left(x_{j-1}\right) .
$$

On the event $\left\{\tau_{0}>\tau_{n}^{\prime}\right\}$, the lower bound $x_{j} \geq a_{0}, 1 \leq j \leq n$ holds and thus $\sum_{j=1}^{n} \chi_{j} \geq$ $n \mu_{-}+A\left(\gamma a_{0}\right)-A\left(x_{0}\right)$. If $I(x)=x-1-\ln x, x>0$, denotes the large deviations rate function for the i.i.d. exponentials $\left(\chi_{j}\right)$, then

$$
P_{x}\left(\tau_{0}>\tau_{n}^{\prime}\right) \leq \exp \left\{-n I\left(\frac{\mu_{-}+1}{2}\right)\right\}, \quad n \geq n\left(x_{0}\right)=\frac{A\left(\gamma a_{0}\right)-A\left(x_{0}\right)}{\mu_{-}-1} .
$$

Using the Borel - Cantelli lemma this proves that $\tau_{0}$ is reached within a finite number of jumps, with probability one. Moreover, the number of jumps necessary to reach $a^{\prime}$ has a finite exponential moment. Since $\tau_{n}^{\prime}<+\infty$ a.s., we proved that $\tau_{0}$ is finite a.s..

Step 2. Let $0<c \leq a^{\prime}$ and $c^{\prime}>a^{\prime}=\gamma^{-1} a_{0}$. We choose a number $w>0$ such that $\gamma\left(c^{\prime}+w\right) \leq \frac{1+\gamma}{2} c^{\prime}$. This will ensure that if $x_{n-1} \leq c^{\prime}$, then $x_{n} \leq \frac{1+\gamma}{2} c^{\prime}$ as soon as $w_{n}^{\prime} \leq w$. Notice that we need at most a finite number of jumps $n^{\prime}=n^{\prime}\left(c, c^{\prime}\right)$ such that $\left(\frac{1+\gamma}{2}\right)^{n^{\prime}} c^{\prime}<c$. We want to prove a lower bound of $P_{x}\left(\tau_{(0, c)} \leq \tau_{n^{\prime}}^{\prime}\right)$, uniform in $x \in\left[c, c^{\prime}\right]$. A lower bound is given by the probability of the event that all $n \leq n^{\prime}$ jumps before hitting $(0, c)$ are occurring after at most $w$ waiting time. Due to the choice of $w$, the process has to stay in $\left[c, c^{\prime}\right]$ in the meantime. If $x \in\left[c, c^{\prime}\right]$ and $\chi$ denotes a mean one exponential r.v., each such event has probability equal to $P_{x}(A(x+w)-A(x)>\chi)$, which is bounded below by the probability of the sub-event $\left\{\chi<w \alpha_{0}\left(\left[c, c^{\prime}\right]\right)\right\}$, where $\alpha_{0}(D)$ denotes the infimum of $\alpha(z)$ on the set $D$. This has positive probability $1-\exp \left(-w \alpha_{0}\left(\left[c, c^{\prime}\right]\right)\right)>0$, independent of $x \in\left[c, c^{\prime}\right]$.

An application of the strong Markov property for each consecutive jump gives the lower bound

$$
P_{x}\left(\tau_{(0, c)} \leq \tau_{n^{\prime}}^{\prime}\right) \geq\left(1-\exp \left(-w \alpha_{0}\left(\left[c, c^{\prime}\right]\right)\right)\right)^{n^{\prime}}=p_{0}>0
$$

independent of $x$. Denote it by $p_{0}$.

Since $P_{x}\left(\tau_{0}=\tau_{\left(0, c^{\prime}\right)}<\infty\right)=1$ for all $x \in(0, \infty)$ from Step 1, the strong Markov property shows that the number of jumps needed to reach $(0, c)$ is stochastically dominated by a geometric r.v. with probability of success $p_{0}$. This shows that $\tau_{(0, c)}$ occurs after a finite number of jumps with probability one. Finally, since $\tau_{n}^{\prime}<+\infty$ a.s., we proved that $P_{x}\left(\tau_{(0, c)}<\infty\right)=1$, for all $x \in(0, \infty)$.

Step 3. 
Without loss of generality, we assume that $A$ is an open interval $(a, b)$ with $0<a<b<$ $\infty$. If $x<a$, there is nothing to prove. Otherwise, pick $c<\min \{a, x\}=x$. We know that $P_{x}\left(\tau_{(0, c)}<\infty\right)=1$.

Let $w^{\prime}$ be the first holding time right after $\tau_{(0, c)}$. Then

$$
\begin{gathered}
P_{x}\left(\tau_{(a, b)}<\infty\right) \geq P_{x}\left(\tau_{(0, c)}<\infty, w^{\prime}>b\right) \geq E_{x}\left[P_{x\left(\tau_{(0, c)}\right)}\left(w^{\prime}>b\right) \mathbf{1}_{\tau_{(0, c)}<\infty}\right] \\
\geq E_{x}\left[\exp \left(-A\left(x\left(\tau_{(0, c)}\right)+b\right)+A\left(x\left(\tau_{(0, c)}\right)\right)\right] \geq \exp (-A(b+c)+A(\gamma c))>0 .\right.
\end{gathered}
$$

Proposition 2. Assume conditions (1.5), (1.8) and (1.7) are satisfied. Let $A$ be a Borel set on $(0, \infty)$ with $l(A)>0$. Then, for any $x \in(0, \infty), P_{x}\left(\int_{0}^{\infty} \mathbf{1}_{A}\left(x_{t}\right) d t=+\infty\right)=1$.

Proof. In analogue manner to the proof of Proposition 1, when (1.7) is satisfied, the chain $\left(x_{n}\right)$, when starting at $x_{0}<\gamma b_{0}$, is bounded below by a random walk that will reach above $\gamma b_{0}$ with probability one. This implies that the process $\left(x_{t}\right)$ reaches above $\gamma b_{0}$ with probability one. By construction, the only way backwards is by performing jumps and the only way forwards is by continuous path motion. It then follows that the process must reach, with probability one, any interval in $\left[x_{0}, \gamma b_{0}\right)$.

Since $l(A)>0$, there exists a compact set $K \subseteq A$ with $l(K)>0$ and $0<a<b<\infty$ such that $K \subseteq(a, b)$. Pick $c<a$ such that $2 c<\min \left\{a, \gamma b_{0}\right\}$.

According to Proposition 1 , the process reaches $(0, c)$ a.s. and since $c<2 c<\gamma b_{0}$, the first paragraph from this proof, together with the strong Markov property applied at the first hitting time of $(0, c)$, show that from any starting point $x$, the process will reach the interval $[c, 2 c)$ with probability one.

Step 1.

Starting with $\tau_{0}^{\prime \prime}=\tau_{[c, 2 c)}$, for $i=0,1,2, \ldots$ we set

$$
\sigma_{i}^{\prime \prime}=\inf \left\{t>\tau_{i}^{\prime \prime} \mid x(t) \geq \gamma b_{0}\right\}, \quad \tau_{i+1}^{\prime \prime}=\inf \left\{t>\tau_{i}^{\prime \prime} \mid x(t) \in[c, 2 c)\right\},
$$

with the convention that the infimum over the empty set equals $+\infty$. The sequence $\left(\tau_{i}^{\prime \prime}\right)$ is strictly increasing almost surely. Let $\tau_{c, i}^{\prime}$ the first jump after $\tau_{i}^{\prime \prime}$. To say that $(a, b)$ is never reached, we must have that $\tau_{c, i}^{\prime}-\tau_{i}^{\prime \prime}=w_{c, i}^{\prime} \leq a$ for all $i$ (any value greater than $a-c$ would be sufficient). This implies

$$
P\left(\inf \left\{t>\tau_{0}^{\prime \prime} \mid x(t) \in(a, b)\right\}=+\infty\right) \leq E\left[\Pi_{i=1}^{\infty} P_{x\left(\tau_{i}^{\prime \prime}\right)}\left(w_{c, i}^{\prime} \leq a\right)\right],
$$


where we applied the strong Markov property for the right-hand side.

Since $x\left(\tau_{i}^{\prime \prime}\right) \in[c, 2 c)$, we may consider the uniform upper bound

$$
\begin{gathered}
P_{x^{\prime}}\left(w_{c, i}^{\prime} \leq a\right)=P\left(A\left(x^{\prime}+a\right)-A\left(x^{\prime}\right)>\chi_{c, i}\right) \\
\leq \sup _{x^{\prime} \in[c, 2 c)}\left\{1-e^{-\left(A\left(x^{\prime}+a\right)-A\left(x^{\prime}\right)\right)}\right\} \leq 1-e^{-\int_{c}^{a+2 c} \alpha(z) d z}<1,
\end{gathered}
$$

where $\chi_{c, i}$ is an exponential random variable of intensity one.

This proves that we can reach $(a, b)$ from $[c, 2 c)$ almost surely.

Step 2.

Again, let $\tau_{0}=\tau_{[c, 2 c)}$. For $i=0,1,2, \ldots$ we set

$$
\sigma_{i}=\inf \left\{t>\tau_{i} \mid x(t) \in(a, b)\right\}, \quad \tau_{i+1}=\inf \left\{t>\sigma_{i} \mid x(t) \in[c, 2 c)\right\},
$$

with the convention that the infimum over the empty set equals $+\infty$. In view of the results from the first paragraph of this proof, the event that all $\tau_{i+1}-\sigma_{i}<\infty, i \geq 0$, has probability one. We notice that for $i \geq 1, \tau_{i+1}-\tau_{i}>a-2 c>0$, bounded below uniformly in $i$, showing that $\lim _{n \rightarrow \infty} \tau_{i}=+\infty$ with probability one.

In addition, Step 1 of the proof showed that $\sigma_{i}-\tau_{i}<\infty$ almost surely (for $i=0$ and any other $i$ ).

To finalize the proof, we observe that

$$
\left\{\int_{\tau_{0}}^{\infty} \mathbf{1}_{A}(x(t)) d t=+\infty\right\} \supseteq\left\{\sum_{i=0}^{\infty} \int_{\tau_{i}}^{\tau_{i+1}} \mathbf{1}_{A}(x(t)) d t=+\infty\right\}
$$

The last event includes $U_{i}-i . o$. , where $U_{i}$ is the event that the first jump after each $\tau_{i}$, with initial position $y=x\left(\tau_{i}\right) \in[c, 2 c)$, occurs at $\tau_{i}+w^{\prime}$, where $w^{\prime}>b-y$. Since these inclusions provide a lower bound for the original event in (3.5), we want to show that $P_{x}\left(U_{i}-i . o.\right)=1$. The complement will have zero probability if

$$
\sup _{y \in[c, a]} P_{y}\left(w^{\prime} \leq b-y\right)=\sup _{y \in[c, a]}\left\{1-e^{-(A(b)-A(y))}\right\} \leq 1-e^{-\int_{c}^{b} \alpha(z) d z}<1
$$

in similar fashion to (3.3). It is important to notice that integrability at zero is not required. 


\section{Small sets}

Throughout this section, we assume (1.5), (1.8), and (1.6)(i) are satisfied. Due to (1.6)(i) we shall assume $A(0+)=0$ in this section. The next Proposition identifies a class of attractive small sets (see Section 6).

Proposition 3. Any set $F=\left(0, a^{\prime}\right]$ with $a^{\prime}>0$ is an attractive small set. More precisely, $P_{x}\left(\tau_{F}<\infty\right)=1$ for all $x \in(0, \infty)$ and there exists $t>0$ and a closed interval $I$ with $I \cap F \neq \emptyset$ such that the probability density component $\rho(t, x, y)$ of $P_{x}(x(t) \in d y)$ is bounded away from zero on $I$, uniformly in $x \in F$.

Remark. It is immediate that $I$ in the proposition is also a small set.

Proof. We shall use the same notations for the holding times and jump times as in the proof of Propositions 1, 2 and Theorem 2. In the proof of Proposition 1 we showed that $P_{x}\left(\tau_{\left(0, a^{\prime}\right)}<\infty\right)=1$ for all $x \in(0, \infty)$. Having $\tau_{\left(0, a^{\prime}\right]} \leq \tau_{\left(0, a^{\prime}\right)}$, condition (i) in Definition 1 in Section 6 is satisfied for any set $\left(0, a^{\prime}\right]$.

Fix $t>0$ such that $t / a^{\prime} \in\left(\gamma^{n}(1-\gamma)^{-1},\left(1-\gamma^{n}\right) \gamma^{-1}\right)$. The interval is properly defined if $\gamma^{n}<1-\gamma$, which is true for sufficiently large $n$. By construction, there exist two numbers $t_{2}>t_{1}>0$ such that $t_{1} \geq \gamma^{n} a^{\prime}+\gamma t$ and $t_{2}<t$ (the second inequality has to be strict) and $I=\left[t_{1}, t_{2}\right] \cap F \neq \emptyset$. A possible choice is $t_{1}=\gamma^{n} a^{\prime}+\gamma t$ and $t_{2}=t-\epsilon$ with small $\epsilon$. Consider $q_{1}<q_{2}$ in $I$.

Pick a point $x \in F=\left(0, a^{\prime}\right]$. We recall that $\tau_{n}^{\prime}=\sum_{j=1}^{n} w_{j}^{\prime}$ is the $n$-th jump time (1.2).

A lower bound of the probability that $x(t)$ falls in the interval $\left(q_{1}, q_{2}\right]$ is

$$
P_{x}\left(q_{1}<x(t) \leq q_{2}\right) \geq P_{x}\left(q_{1}<x(t) \leq q_{2}, \tau_{n}^{\prime} \leq t<\tau_{n+1}^{\prime}\right)
$$

by intersection with the event that there were exactly $n$ jumps up to time $t$. Writing $x(t)=x\left(\tau_{n}^{\prime}\right)+\left(t-\tau_{n}^{\prime}\right)$, using formula (1.4) which says that $x\left(\tau_{n}^{\prime}\right)=\gamma^{n} x+\sum_{j=1}^{n} \gamma^{n+1-j} w_{j}^{\prime}$, the event on the right hand side of (4.1) is the intersection of

$$
G_{n}=\left\{q_{1}<\gamma^{n} x+\sum_{j=1}^{n} \gamma^{n+1-j} w_{j}^{\prime}+\left(t-\sum_{j=1}^{n} w_{j}^{\prime}\right) \leq q_{2}\right\}
$$


and $R_{n}=\left\{\sum_{j=1}^{n} w_{j}^{\prime} \leq t<\sum_{j=1}^{n+1} w_{j}^{\prime}\right\}$. The choice of the interval $I$ makes the inequality $\sum_{j=1}^{n} w_{j}^{\prime} \leq t$ redundant. To see that, we re-write the left hand side of (4.2)

$$
t>\sum_{j=1}^{n}\left(1-\gamma^{n+1-j}\right) w_{j}^{\prime}+q_{1}-\gamma^{n} x \geq(1-\gamma) \sum_{j=1}^{n} w_{j}^{\prime}+\left(\gamma^{n} a^{\prime}+\gamma t\right)-\gamma^{n} a^{\prime}
$$

and simplify. The remaining condition $t<\sum_{j=1}^{n+1} w_{j}^{\prime}$, written as $w_{n+1}^{\prime}>t-\sum_{j=1}^{n} w_{j}^{\prime}$, is equivalent to

$$
\chi_{n+1}>A\left(x\left(\tau_{n}^{\prime}\right)+t-\sum_{j=1}^{n} w_{j}^{\prime}\right)-A\left(x\left(\tau_{n}^{\prime}\right)\right)
$$

Denote

$$
Y_{n}=\sum_{j=1}^{n}\left(1-\gamma^{n+1-j}\right) w_{j}^{\prime} .
$$

We note that the random variables $Y_{j}, j=1,2, \ldots$ also depend on the initial point $x$. Inductively, from (4.9), one can see that the vector $w^{\prime}=\left(w_{1}^{\prime}, \ldots, w_{n}^{\prime}\right)$, and all $\tau_{i}^{\prime}, 1 \leq$ $i \leq n$ are given as deterministic functions of $\chi=\left(\chi_{1}, \ldots, \chi_{n}\right)$. This implies that $\chi_{n+1}$ is independent of $w^{\prime}=\left(w_{1}^{\prime}, \ldots, w_{n}^{\prime}\right)$.

Let's denote the density functions of $w^{\prime}=\left(w_{1}^{\prime}, \ldots, w_{n}^{\prime}\right)$ and $Y_{n}$ by $\rho_{n}\left(w^{\prime}\right)$, respectively $g_{n}\left(y_{n}\right)$. The existence of these densities is proven in Propositions 4 and 5 .

We recall that $G_{n}=\left\{q_{1}<t+\gamma^{n} x-Y_{n} \leq q_{2}\right\}$. On the interval $I$, the lower bound (4.1) can be written as

$$
\int_{G_{n}} \exp \left\{-\left(A\left(x\left(\tau_{n}^{\prime}\right)+t-\sum_{j=1}^{n} w_{j}^{\prime}\right)-A\left(x\left(\tau_{n}^{\prime}\right)\right)\right)\right\} \rho_{n}\left(w^{\prime}\right) d w^{\prime} .
$$

On the event $G_{n}, x(t)=x\left(\tau_{n}^{\prime}\right)+t-\sum_{j=1}^{n} w_{j}^{\prime}<q_{2} \leq t_{2}$. Then the exponential factor is bounded below by $\exp \left(-A\left(t_{2}\right)\right)$. Then (4.5) has lower bound

$$
\exp \left(-A\left(t_{2}\right)\right) \int_{G_{n}} \rho_{n}\left(w^{\prime}\right) d w^{\prime}=\exp \left(-A\left(t_{2}\right)\right) \int_{-q_{2}+\gamma^{n} x+t}^{-q_{1}+\gamma^{n} x+t} g_{n}\left(y_{n}\right) d y_{n} .
$$

Due to the choice of $t_{1}$ and $t_{2},-q_{2}+\gamma^{n} x+t>t-t_{2}>0$ and $-q_{1}+\gamma^{n} x+t \leq t(1-\gamma)$, independently of $x$. Let's choose $b>0$ from Proposition 5 as $b=\max \left\{a^{\prime}, t_{2}, t(1-\gamma)\right\}$. In that case let $d_{n}=\inf _{y_{n} \in\left[t-t_{2}, t(1-\gamma)\right]} g_{n}\left(y_{n}\right)$, which is necessarily strictly positive from Proposition 5.

Then

$$
P_{x}\left(q_{1}<x(t) \leq q_{2}\right) \geq \exp \left(-A\left(t_{2}\right)\right) d_{n}\left(q_{2}-q_{1}\right)
$$


Inequality (4.7) implies that the interval $I$ is a small set for the process $x(\cdot)$, by taking the lower bound probability measure equal to the uniform (normalized Lebesgue) measure on I.

It remains to prove that $g_{n}\left(y_{n}\right)$ exists and that $d_{n}>0$ does not depend on $x$, which is done in Proposition 5.

Proposition 4. The probability distribution of $x(t)$ has a singular component before the first jump and an absolutely continuous component after the first jump. More precisely, if $x \in(0, \infty)$

$$
P_{x}(x(t) \in d y)=P_{x}\left(\tau_{1}^{\prime}>t\right) \delta_{x+t}(d y)+\rho(t, x, y) d y
$$

where

$$
\rho(t, x, y)=\sum_{n=1}^{\infty} \rho_{n}(t, x, y), \quad \rho_{n}(t, x, y)=\frac{d}{d y} P_{x}\left(x(t) \in d y, \tau_{n}^{\prime} \leq t<\tau_{n+1}^{\prime}\right) .
$$

Proof. The first term of the formula is due to the uniform deterministic motion at speed +1 until the first jump. Let $A$ a measurable null set with respect to the Lebesgue measure $l(\cdot)$. To prove the absolute continuity with respect to the Lebesgue measure of $P_{x}(x(t) \in$ $\left.d y, \tau_{n}^{\prime} \leq t<\tau_{n+1}^{\prime}\right), n \geq 1$, in view of (1.4), we have the formula

$$
x(t)=x\left(\tau_{n}^{\prime}\right)+t-\tau_{n}^{\prime}=t+\gamma^{n} x-\sum_{k=0}^{n-1}\left(1-\gamma^{k+1}\right) w_{n-k}^{\prime} .
$$

when there are exactly $n$ jumps before time $t>0$ where $n=1, \ldots$

Denote $w^{\prime}=\left(w_{1}^{\prime}, \ldots, w_{n}^{\prime}\right)$ and $\ell: \mathbb{R}^{n} \rightarrow \mathbb{R}^{n}$ the invertible linear map with components $\ell_{1}\left(w^{\prime}\right)=-\sum_{k=0}^{n-1}\left(1-\gamma^{k+1}\right) w_{n-k}^{\prime}, \ell_{i}\left(w^{\prime}\right)=w_{i}^{\prime}, 2 \leq i \leq n$ (if $n=1$ only $\ell_{1}$ is needed).

Then, if $A_{n}=A-t-\gamma^{n} x$, evidently with $l\left(A_{n}\right)=0$

$$
\begin{aligned}
P_{x}(x(t) \in & \left.A, \tau_{n}^{\prime} \leq t<\tau_{n+1}^{\prime}\right)=P_{x}\left(\ell_{1}\left(w_{1}^{\prime}, \ldots, w_{n}^{\prime}\right) \in A_{n}, \sum_{k=1}^{n} w_{k}^{\prime} \leq t<\sum_{k=1}^{n+1} w_{k}^{\prime}\right) \\
& \leq P_{x}\left(\ell_{1}\left(w_{1}^{\prime}, \ldots, w_{n}^{\prime}\right) \in A_{n}\right)=P_{x}\left(\ell\left(w^{\prime}\right) \in A_{n} \times \mathbb{R}^{n-1}\right)=0
\end{aligned}
$$

as soon as $w^{\prime}$ has a density, which is shown in Proposition 5. The summation $\rho=\sum_{n=1}^{\infty} \rho_{n}$ is an integrable function, and thus a density, an immediate fact from the monotone convergence theorem.

The proof of Proposition 5 needs the following lemma. 
Lemma 1. Let $V$ be a $d$-dimensional random variable and $W$ be a one dimensional nonnegative random variable, with joint density $f(v, w)$ having the property that for any $b>0$, there exists a function $k_{1}(w)=k_{1}^{b}(w)$ such that $f(w \mid V=v) \geq k_{1}(w)$ for all $v \in \mathbb{R}^{d}$ and $w \in(0, b]$. Let $F(v)$ be a nonnegative measurable function. Then, for all $y \in(0, b]$, the density function $f_{Y}$ of $Y=F(V)+W$ satisfies

$$
f_{Y}(y) \geq \int_{F(v) \leq y} k_{1}(y-F(v)) f_{V}(v) d v .
$$

Proof. First we fix $b>0$. Then for any $y \in(0, b]$,

$$
\begin{gathered}
f_{Y}(y)=\int_{F(v) \leq y} f(v, y-F(v)) d v= \\
\int_{F(v) \leq y} f(y-F(v) \mid V=v) f_{V}(v) d v \geq \int_{F(v) \leq y} k_{1}(y-F(v)) f_{V}(v) d v,
\end{gathered}
$$

where we used the fact that both $F(v)$ and $W$ are nonnegative implies that $y-F(v)$ belongs to $(0, b]$.

Proposition 5. The random variables $w^{\prime}=\left(w_{1}^{\prime}, \ldots, w_{n}^{\prime}\right)$ have a strictly positive density $h_{n}\left(w^{\prime}\right)$ depending on the initial point $x$ and $n$ only. For any $b>0$, the random variables $Y_{n}$ defined in (4.4) have density $g_{n}\left(y_{n}\right)$ with the property that for any $x \in(0, b]$ and any $y_{n} \in(0, b]$, there exists a bounded function $c_{n}^{b}(\cdot)$ independent of $x$, bounded away from zero on compact sets, such that $g_{n}\left(y_{n}\right) \geq c_{n}^{b}\left(y_{n}\right)$.

Proof. Step 1. First, we show that $w^{\prime}=\left(w_{1}^{\prime}, \ldots, w_{n}^{\prime}\right)$ has a density for all $n \geq 1$. In general, for a Borel set $G$ in $(0, \infty)^{n}$ and $w>0$

$$
P_{x}\left(w_{n+1}^{\prime}>w, w^{\prime} \in G\right)=\int_{G} P_{x}\left(w_{n+1}^{\prime}>w \mid w^{\prime}=v\right) P_{x}\left(w^{\prime} \in d v\right)
$$

so it is sufficient to prove that $P_{x}\left(w_{n+1}^{\prime}>w \mid w^{\prime}=v\right)$ is absolutely continuous with density $\rho(w \mid v)$, both for the verification step and the induction step.

From (1.4) we see that $w_{n+1}^{\prime}$ depends on the value $v$ of the vector $w^{\prime}$ via the number $x(v)=\gamma^{n} x+\sum_{k=0}^{n} \gamma^{k+1} v_{n-k}$. We have the exact formula for the density $\rho(w \mid v)$

$$
P_{x}\left(w_{n+1}^{\prime}>w \mid w^{\prime}=v\right)=P_{x(v)}\left(A(x(v)+w)-A(x(v))<\chi_{n+1}\right)=e^{-(A(x(v)+w)-A(x(v)))}
$$

$$
\rho(w \mid v)=e^{-(A(x(v)+w)-A(x(v)))} \alpha(x(v)+w) .
$$


As long as $A(+\infty)=+\infty$ this is a proper distribution function. It is always positive because $A$ is finite and $\alpha$ is bounded away from zero on any compact set.

Step 2. We first show that on the event $\left\{0<\sum_{i=1}^{n} w_{i}^{\prime} \leq b(1-\gamma)^{-1}\right\}, h_{n}(\cdot)$ is bounded below by a product function independent of $x$.

Since $x \leq b$ and $Y_{n} \leq b$, then

$$
x\left(\tau_{k}^{\prime}\right) \leq \gamma b\left(1+(1-\gamma)^{-1}\right)=M(b), \quad 0 \leq k \leq n .
$$

In this case, each density (4.13) is bounded below for all $w>0$ by

$$
e^{-(A(x(v)+w)-A(x(v)))} \alpha(x(v)+w) \geq e^{-A(M(b)+w)} \alpha_{0}([w, w+M(b)])=\rho_{-}(w)>0, \quad w>0 .
$$

The lower bound is bounded away from zero on compact sets; otherwise the continuous function $\alpha(\cdot)$ would have a zero on a compact set, which is impossible. It is also integrable on compact sets, being dominated by $\alpha(w+b)$.

We proceed by induction over $n$. The verification step is given in (4.15). Suppose it is true for $n-1$.

From (4.11) and (4.13), combined with the fact that if $\sum_{i=1}^{n} w_{i}^{\prime} \leq b(1-\gamma)^{-1}$ then a fortiori $\sum_{i=1}^{n-1} w_{i}^{\prime} \leq b(1-\gamma)^{-1}$, we obtain that

$$
\begin{gathered}
h_{n}\left(w_{1}^{\prime}, \ldots, w_{n-1}^{\prime}, w_{n}^{\prime}\right)=\rho\left(w_{n}^{\prime} \mid w_{1}^{\prime}, \ldots, w_{n-1}^{\prime}\right) h_{n-1}\left(w_{1}^{\prime}, \ldots, w_{n-1}^{\prime}\right) \\
\geq \rho_{-}\left(w_{n}^{\prime}\right) \Pi_{i=1}^{n-1} \rho_{-}\left(w_{i}^{\prime}\right), \quad \sum_{i=1}^{n} w_{i}^{\prime} \leq b(1-\gamma)^{-1} .
\end{gathered}
$$

Step 3. Since $(1-\gamma) \sum_{i=1}^{n} w_{i}^{\prime} \leq Y_{n} \leq \sum_{i=1}^{n} w_{i}^{\prime}$, it follows that

$$
\left\{0<Y_{n} \leq b\right\} \subseteq\left\{0<\sum_{i=1}^{n} w_{i}^{\prime} \leq b(1-\gamma)^{-1}\right\}
$$

and thus the lower bound will be satisfied when $Y_{n} \leq b$.

We prove by induction the lower bound on the density $g_{n}\left(y_{n}\right)$ of $Y_{n}$.

The verification step is immediate since $Y_{1}=(1-\gamma) w_{1}^{\prime}$. We set

$$
c_{1}^{b}(w)=(1-\gamma)^{-1} \rho_{-}\left(w(1-\gamma)^{-1}\right) .
$$

Assuming the statement is true for $n-1$, we prove it for $n$. We use Lemma 1 with write $Y_{n}=F(V)+W$ with $V=w^{\prime}, F(v)=\sum_{j=1}^{n-1}\left(1-\gamma^{n+1-j}\right) v_{j}$ and $W=(1-\gamma) w_{n}^{\prime}$. The pair $(V, W)$ satisfies Lemma 1 with $Y=Y_{n}$. 
Due to (4.14), we can set $k_{1}(w)=c_{1}^{b}(w)$, where $k_{1}(w)$ is as in Lemma 1. By the induction hypothesis, we obtain

$$
\begin{gathered}
g_{n}\left(y_{n}\right) \geq \int_{F(v) \leq y_{n}} c_{1}^{b}\left(y_{n}-F(v)\right) h_{n-1}(v) d v \geq \\
\int_{F(v) \leq y_{n}} c_{1}^{b}\left(y_{n}-F(v)\right) \Pi_{i=1}^{n-1} \rho_{-}\left(v_{i}\right) d v=c_{n}^{b}\left(y_{n}\right)>0 \quad y_{n} \in(0, b] .
\end{gathered}
$$

We want to show that $g_{n}\left(y_{n}\right)$ is bounded on $[\epsilon, b]$, a compact interval included in $(0, b]$. A lower bound can be obtained from the last inequality by integrationg over the set $\{\epsilon \leq$ $\left.F(v) \leq y_{n}\right\}$. By construction (4.15), the function $c_{1}^{b}(\cdot)$ is bounded away from zero on compact sets. Since $\int_{\epsilon \leq F(v) \leq y_{n}} \Pi_{i=1}^{n-1} \rho_{-}\left(v_{i}\right) d v>0$ (convolution of independent positive r.v.), we are done.

Proposition 6. There exists $t_{0} \geq 0$ and $F$ a small set with $l(F)>0$ such that $P_{x}(x(t) \in$ $F)>0$ for all $t \geq t_{0}$ and all $x \in F$ and hence the process $x(t)$ is $l$-aperiodic.

Proof. Let $F=\left(0, a^{\prime}\right]$ as in Proposition 3. Let $t>0$ be arbitrary. We shall construct the event

$$
A_{t}=\left\{\gamma\left(a^{\prime}+w_{1}^{\prime}\right)<c a^{\prime}\right\} \cap\left(\cap_{i=2}^{N}\left\{\epsilon_{1}<w_{i}^{\prime}<\epsilon_{2}\right\}\right)
$$

where $c$ is a number in $(\gamma, 1), \epsilon_{2}=a^{\prime}(1-c), \epsilon_{1} \in\left(0, \epsilon_{2}\right)$ and $N=\left[t / \epsilon_{1}\right]+2$. Set $t_{0}=$ $\left(c \gamma^{-1}-1\right) a^{\prime}$. Under $A_{t}$, the choice of $t_{0}$ ensures that the first jump occurs before $t_{0}$ and brings $x(t)$ below $c a^{\prime}<a^{\prime}$. The choice of the constant $c$ ensures that both $\gamma\left(c a^{\prime}+w_{i}^{\prime}\right)<c a^{\prime}$ (the process returns to a point in $\left(0, c a^{\prime}\right)$ after each jump) and $c a^{\prime}+w_{i}^{\prime}<a^{\prime}$ (the process will not exceed $a^{\prime}$ ) for the next $N-1$ steps. The lower bound $\epsilon_{1}$ is arbitrary except that the corresponding $N$ must be greater or equal to one. By choosing an intersection over the first $N$ jumps we make sure that when $x(t)$ starts at $x(0)=x \leq a^{\prime}$ there can be at most $N$ jumps in the time interval $[0, t]$ and thus $x(t)$ stays in $\left(0, a^{\prime}\right]$ on $\left[t_{0}, t\right]$, hence $x(t) \in F$.

The event $A_{t}$ is can be evaluated as a function of $w^{\prime}=\left(w_{1}^{\prime}, \ldots, w_{N}^{\prime}\right)$, more precisely $A_{t}=\left\{w^{\prime} \in\left(0, t_{0}\right) \times\left(\epsilon_{1}, \epsilon_{2}\right)^{N-1}\right\}$, a set in $(0, \infty)^{N}$ with positive probability. Since $w^{\prime}$ has positive density from Proposition 5 and $\{x(t) \in F\} \supset A_{t}$, for any $x \in F$, we have $P_{x}(x(t) \in F) \geq P_{x}\left(A_{t}\right)>0$, proving that $x(t)$ is aperiodic. 


\section{Speed of convergence to equilibrium}

In the preceding sections we proved that any $F=\left(0, a^{\prime}\right], a^{\prime}>0$ is an attractive small set.

For $\delta>0$, let $\tau_{F}(\delta)=\inf \{t \geq \delta \mid x(t) \in F\}$ be the first time of return to the set $F$ (see also Section 6). Consider a nondecreasing function $B(t), t \geq 0, B(t) \geq 0$. We are interested in showing that there exists $\delta>0$ such that the $B$-moment of $\tau_{F}(\delta)$ is uniformly bounded over any starting point in $F$, i.e.

$$
\sup _{x \in F} E_{x}\left[B\left(\tau_{F}(\delta)\right)\right]<\infty .
$$

Due to the form of $F=\left(0, a^{\prime}\right]$ and the pathwise bound

$$
x(t) \leq x+t, \quad x(0)=x,
$$

it is sufficient to allow $x \in\left(0, a^{\prime}+\delta\right]$ and simply prove the bound

$$
\sup _{x \in(0, b]} E_{x}\left[B\left(\tau_{\left(0, a^{\prime}\right]}\right)\right]<\infty, \quad \forall b>a^{\prime} .
$$

Lemma 2. Let $f(t, x)$ continuously differentiable in $t \geq 0, x>0$, nondecreasing in both $t$ and $x$ such that there exists $t_{0} \geq 0$ satisfying

$$
\frac{\partial}{\partial t} f(t, x(t))+\frac{\partial}{\partial x} f(t, x(t))+\alpha(x(t))(f(t, \gamma x(t))-f(t, x(t))) \leq 0
$$

for any $t \geq t_{0}$, then

$$
E_{x}\left[f\left(\tau_{\left(0, a^{\prime}\right]}, \gamma a^{\prime}\right) \mathbf{1}_{\left\{\tau_{\left(0, a^{\prime}\right]} \geq t_{0}\right\}}\right] \leq f\left(t_{0}, x+t_{0}\right) .
$$

In particluar, if $f(t, x)=B(t) R(x)$, then (5.3) is satisfied.

Proof. We first verify that $E_{x}[f(t, x(t))] \leq f(t, x+t)<\infty$. Writing Ito's formula for $f(t, x(t)), t \geq t_{0}$ we obtain from (5.4) that $f(t, x(t)), t \geq t_{0}$ is a supermartingale. After stopping at $\tau_{\left(0, a^{\prime}\right]} \vee t_{0}$ - recall that $\tau_{\left(0, a^{\prime}\right]}<\infty$ a.s. - we have the inequality

$$
E_{x}\left[f\left(\tau_{\left(0, a^{\prime}\right]} \vee t_{0}, x\left(\tau_{\left(0, a^{\prime}\right]} \vee t_{0}\right)\right)\right] \leq E_{x}\left[f\left(t_{0}, x\left(t_{0}\right)\right)\right] \leq f\left(t_{0}, x+t_{0}\right) .
$$

At $\tau_{\left(0, a^{\prime}\right]}$, the process has just re-entered $\left(0, a^{\prime}\right]$, meaning that it jumped from $\gamma^{-1} x\left(\tau_{\left(0, a^{\prime}\right]}\right)=$ $x\left(\tau_{\left(0, a^{\prime}\right]}-\right)>a^{\prime}$ and thus $x\left(\tau_{\left(0, a^{\prime}\right]}\right) \geq \gamma a^{\prime}$. To make sure $\tau_{\left(0, a^{\prime}\right]}$ is achieved, we insert the indicator function $\mathbf{1}_{\left\{\tau_{\left(0, a^{\prime}\right\}} \geq t_{0}\right\}}$, which bounds below the left-hand side

$$
E_{x}\left[f\left(\tau_{\left(0, a^{\prime}\right]}, \gamma a^{\prime}\right) \mathbf{1}_{\left\{\tau_{\left(0, a^{\prime}\right]} \geq t_{0}\right\}}\right] \leq E_{x}\left[f\left(\tau_{\left(0, a^{\prime}\right]} \vee t_{0}, x\left(\tau_{\left(0, a^{\prime}\right]} \vee t_{0}\right)\right)\right]
$$


which proves (5.5). When $f(t, x)=B(t) R(x)$, inequality (5.5) proves that the $B$-moment of $\tau_{\left(0, a^{\prime}\right]}$ is finite, and the right-hand side is uniformly bounded over $x \in(0, b]$.

Recalling the definition (1.2) of the $j$-th holding time $w_{j}^{\prime}=\tau_{j}^{\prime}-\tau_{j-1}^{\prime}, j \geq 1$, we calculate

$$
P_{x}\left(w_{1}^{\prime}>w\right)=P_{x}\left(A(x+w)-A(x)<\chi_{1}\right)=e^{-(A(x+w)-A(x))} .
$$

Proposition 7. Assume there exists $\beta>0, a_{1}>0$ such that $x \alpha(x) \geq \beta$ for any $x \geq a_{1}$. If $\beta$ is sufficiently large, the first moment of $\tau_{\left(0, a^{\prime}\right]}$ is uniformly bounded, i.e. (5.3) is true for $B(t)=t$.

Proof. Inequality (5.4) applied to $f(t, x)=t x^{m}, m>0$ can be re-written as

$$
t x(t)^{m-1}\left[\frac{x(t)}{t}+m-\beta\left(1-\gamma^{m}\right)\right] \leq t x(t)^{m-1}\left[\frac{b+t}{t}+m-\beta\left(1-\gamma^{m}\right)\right] \leq 0
$$

as soon as $t \geq t_{0}=b\left(\beta\left(1-\gamma^{m}\right)-(1+m)\right)^{-1}$. It follows that for any $\beta>\beta^{*}$,

$$
\beta^{*}=\inf _{m>0}(1+m)\left(1-\gamma^{m}\right)^{-1}
$$

the first moment of the time of return is finite.

Proposition 8. Assume there exists $\beta>0, a_{1}>0$ such that $\alpha(x) \geq \beta$ for any $x \geq a_{1}$. Then $\tau_{\left(0, a^{\prime}\right]}$ has uniformly bounded exponential moments, i.e. (5.3) is true for $B(t)=$ $\exp (-\theta t)$, with some $\theta<0$. Moreover, for sufficiently large $a^{\prime}$, the number $\theta$ can be taken arbitrarily close to $-\beta$.

Proof. Inequality (5.4) applied to $f(t, x)=\exp (-\theta t) x^{m}, m>0$ can be re-written as

$$
\begin{aligned}
& \exp (-\theta t) x(t)^{m}\left[-\theta+\frac{m}{x}-\beta\left(1-\gamma^{m}\right)\right] \\
\leq & \exp (-\theta t) x(t)^{m}\left[-\theta+\frac{m}{a^{\prime}}-\beta\left(1-\gamma^{m}\right)\right] .
\end{aligned}
$$

For any fixed $\beta, m$ we shall have $\theta<0$ satisfying the inequality. In order to optimize the range of $\theta$, pick $m$ arbitarily close to zero and $a^{\prime}$ sufficiently large, showing that for $\theta \in(-\beta, \infty)$ the inequality is satisfied. 


\subsection{Proof of Theorem 2.}

Proof. (i) We first show that the Lebesgue measure $l(d x)$ is maximal (see Definition 2 and the discussion thereafter). Given that the process is $l(d x)$ - Harris recurrent from Proposition $2, l(d x)$ is an irreducibility measure. It is sufficient to show that $P_{x}(x(t) \in d y)$ has a density. This is proven in Proposition 4. In that case, if $l(A)=0$ implies that $P_{x}(x(t) \in A)=0$ and so $G(x, A)=0$. If $\nu$ is another irreducibility measure and $\nu(A)>0$, we should have $G(x, A)>0$, a contradiction; thus $\nu<<l$.

The process is non-explosive as shown in Theorem 1. Proposition 2 proves $l(d x)$ - Harris recurrence. Proposition 3 identifies all intervals $\left(0, a^{\prime}\right]$ to be Doeblin attractive sets, under (1.6)(i) - integrability at zero. Proposition 6 proves that $x(t)$ is aperiodic. Proposition 7 shows that the process is positive recurrent, proving (ii). Finally Proposition 8 shows that the conditions of Theorem 6 are satisfied for any $F=\left(0, a^{\prime}\right]$ with some $\delta>0$ and $\eta>0$, proving (iii). Because the reference measure $\nu_{0}$ in Doeblin's theorem is continuous with density bounded away from zero on a compact interval, the assertion that the Doebin set may be taken compact is verified.

\subsection{Proof of Theorem 3.}

Proof. Inequality (5.4) applied to $f(t, x)=\exp \left(t^{1+q}\right) x^{m}, m>0$ can be re-written as

$$
\begin{gathered}
\exp \left(t^{1+q}\right) x(t)^{m+p}\left[(1+q) t^{q} x(t)^{-p}+m x^{-p-1}-\beta\left(1-\gamma^{m}\right)\right] \\
\leq \exp \left(t^{1+q}\right) x(t)^{m+p}\left[(1+q) t^{q}(b+t)^{-p}+m x^{-p-1}-\beta\left(1-\gamma^{m}\right)\right] .
\end{gathered}
$$

As soon as $a^{\prime}$ and $t \geq t_{0}$ are fixed but sufficiently large, the expression on the right hand side is non-positive. Here we used that $x(t) \geq b+t$ and $q \leq p<0$. When $q=p$ it is sufficient to have $\beta$ large enough and the inequality is satisfied.

For the last statement of the proposition, in the special case $\alpha(x)=x^{p}, p \in(-1,0)$ as $x \rightarrow \infty$, we see that $\tau_{\left(0, a^{\prime}\right]}$ has no finite exponential moment simply by calculating $E_{x}\left[w_{1}^{\prime}\right]=+\infty$ as in the proof of Theorem 4 (iii).

\subsection{Proof of Theorem 4.}

Proof. Part a) 
(i) Based on (1.3) and the particular form of $A(x)=\beta \ln x$ when $x \geq a_{1}$,

$$
x_{j}=x \gamma^{n} \exp \left(\beta^{-1} \sum_{i=1}^{j} \chi_{i}\right)
$$

such that $\ln x_{j}, j \geq 0$ is a simple random walk with i.i.d. increments with expectation $\beta^{-1}+\ln \gamma$ stopped upon hitting $\left(-\infty, \ln a_{1}\right]$. It follows that $\lim _{j \rightarrow \infty} x_{j}=+\infty$ with positive probability if $\beta<(-\ln \gamma)^{-1}$ and $\lim _{j \rightarrow \infty} x_{j} \leq \ln a_{1}$ with probability one if $\beta \geq(-\ln \gamma)^{-1}$. The third assertion in (i) is immediate from Proposition 7.

(ii) Let $a^{\prime}>a_{1}$. Using (1.3) and (1.4) we can write

$$
\tau_{\left(0, a^{\prime}\right]}=\sum_{i=1}^{N} w_{i}^{\prime}=\gamma^{-1}\left(H_{N}-x_{0}\right)-H_{N-1}, \quad \forall n \geq 1 \quad H_{n}=\sum_{j=0}^{n} x_{j}
$$

where $N$ is the hitting time of the set $\left(0, a^{\prime}\right]$ by the disctrete time process $\left(x_{n}\right), x_{0}:=x$ of positions right after each jump. From the tail distribution formula (5.6)

$$
E_{x_{i-1}}\left[w_{i}^{\prime}\right]=\int_{0}^{\infty}\left(\frac{x_{i-1}}{x_{i-1}+w}\right)^{\beta} d w=(\beta-1)^{-1} x_{i-1}, \quad i \geq 1
$$

and $E_{x}\left[\tau_{\left(0, a^{\prime}\right]}\right] \leq(\beta-1)^{-1} \sum_{i=1}^{\infty} E_{x}\left[x_{i-1}\right]<+\infty$ as the sum of a geometric series with rate $\beta \gamma(\beta-1)^{-1}<1$. In this case it is easy to verify that $u(x)=E_{x}\left[\tau_{\left(0, a^{\prime}\right]}\right]$ satisfies the equation $B u(x)=-1, u(x)=0$ if $x \leq a_{1}$. The soultion will have two regimes: when $x \leq a^{\prime} \gamma^{-1}$ and $x>a^{\prime} \gamma^{-1}$. On the first interval the equation $-1=B u(x)=u^{\prime}(x)+\alpha(x)(-u(x))$ since the position after the first jump gives $u(\gamma x)=0$. It is immediate that $u(x)$ will be a linear combination of $x^{q}, q=0,1, \beta$.

Moreover, for $x \geq a_{1} / \gamma$ the linear function

$$
u(x)=k_{1} x+k_{2}, \quad \frac{1}{k_{1}}=\beta(1-\gamma)-1
$$

satisfies the equation. The polynomial bound shows that solutions are unique. From the lines above we see that $u(x)$ has a linear bound in $x$, implying uniqueness.

We proceed to give an exact formula. Let $y=x+w^{\prime}$ be the position right before the first jump. We can see that

$$
u(x)=\int_{x}^{\infty}(y-x) \alpha(y) e^{-A(y)+A(x)} d y+\int_{x \vee a^{\prime} \gamma^{-1}}^{\infty} u(\gamma y) \alpha(y) e^{-A(y)+A(x)} d y
$$

which proves continuity in $x$ on $\left[a^{\prime} \gamma^{-1}, \infty\right)$ and the presence of a jump at $a^{\prime} \gamma^{-1}$. In the present case the first integral is equal to $x /(\beta-1)$. 
After some calculations,

$$
u(x)=\left\{\begin{array}{llll}
\frac{x}{\beta-1}+C \beta \gamma^{\beta} x^{\beta}, & \text { if } & x \leq a^{\prime} \gamma^{-1} \\
\frac{x}{\beta(1-\gamma)-1}+D, & \text { if } & x>a^{\prime} \gamma^{-1}
\end{array}\right.
$$

where $C$ and $D$ can be determined from each other by continuity and $C=\int_{a^{\prime}}^{\infty} u(z) z^{-\beta-1} d z$. This formula allows us to obtain $C$ after splitting the integral in two parts, corresponding to the two regimes. Solving the resulting equation,

$$
C=\frac{\beta(1-\gamma)+\gamma^{\beta}}{\left(a^{\prime}\right)^{\beta}(\beta-1)^{2}(\beta(1-\gamma)-1)\left(1+\beta \gamma^{\beta} \ln \gamma-\gamma^{\beta}\right)} .
$$

(iii) Revisiting (5.9) we see that the expected valuee of $\tau_{\left(0, a^{\prime}\right]}$ is finite if and only if $E_{x}\left[H_{N}\right]<\infty$.

Let $\beta^{\prime \prime}>\beta^{\prime}>(1-\gamma)^{-1}$ and write $N_{\beta^{\prime}}, N_{\beta^{\prime \prime}}$, as well as $x_{n}^{\beta^{\prime}}, x_{n}^{\beta^{\prime \prime}}$ for the corresponding chains. The underlying holding times $\left(\chi_{n}\right)$ being the same, we have a robust coupling that allows us to see that $x_{j}^{\beta^{\prime}}>x_{j}^{\beta^{\prime \prime}}$ and $N_{\beta^{\prime}}>N_{\beta^{\prime \prime}}$. Then $\sum_{j=1}^{N_{\beta^{\prime}}} x_{j}^{\beta^{\prime}} \geq \sum_{j=1}^{N_{\beta^{\prime \prime}}} x_{j}^{\beta^{\prime}} \geq \sum_{j=1}^{N_{\beta^{\prime \prime}}} x_{j}^{\beta^{\prime \prime}}$ which proves monotonicity of the expected value (non-increasing) in the parameter $\beta>$ $(1-\gamma)^{-1}$. Using the explicit formula $(5.12)$ we see that $\lim _{\beta \downarrow(1-\gamma)^{-1}} E_{x}\left[\tau_{\left(0, a^{\prime}\right]}\right]=+\infty$.

\section{Part b)}

The proof is essentially the same as in Part a) (i) by evaluating the random walk $\ln \left(x_{j}\right)$, $j \geq 1$ the number of jumps and its drift.

\section{Appendix}

6.1. Local Doeblin theory for continuous time processes. We start with the introduction of the basic concepts relevant to the theory of continuous time Markov processes on general state spaces.

Let $\{x(t)\}_{t \geq 0}$ be a continuous time non - explosive Markov process on the state space $\mathcal{S}$ with Borel sets $\mathcal{B}(\mathcal{S})$. For a Borel set $A, \tau_{A}=\inf \{t \geq 0 \mid x(t) \in A\}$ is the first hitting time of $A$ and if $x \in \mathcal{S}$

$$
G(x, A)=E_{x}\left[\int_{0}^{\infty} \mathbf{1}_{A}(x(t)) d t\right]
$$

denotes the Green function associated to the process.

Definition 1. (Local Doeblin condition and small sets) A Borel subset $F$ in the state space $\mathcal{S}$ of the Markov process will be said 
(i) attractive, if $P_{x}\left(\tau_{F}<\infty\right)=1$, for any $x \in \mathcal{S}$, and

(ii) small, if there exists a time $t>0$, a probability measure $\nu_{0}(d x)$ concentrated on $F$, and a constant $c \in(0,1)$ such that, for all $x \in F$ and all Borel sets $B$ of $\mathcal{S}$, we have $P_{x}(x(t) \in B) \geq c \nu_{0}(B)$.

A set $F$ satisfying (i)-(ii) is also called an attractive Doeblin set.

Definition 2. Given $\phi$ a measure on $(\mathcal{S}, \mathcal{B}(\mathcal{S}))$, a process is said $\phi$-irreducible if for any Borel set $B$ of $\mathcal{S}$ with $\phi(B)>0$, then $G(x, B)>0$ for any $x \in \mathcal{S}$. We also say that $\phi$ is an irreducibility measure. The process is said aperiodic if there exists a small set $F$ with $\phi(F)>0$ and a time $t_{0} \geq 0$ such that $P_{x}(x(t) \in F)>0$ for all $t \geq t_{0}$ and $x \in F$.

We note that whenever there exists $\phi$ as above, there exists a maximal irreducibility measure $\psi$ such that $\nu<<\psi$ for any irreducibility measure $\nu$. Hence aperiodicity can be defined directly in terms of $\psi$; at the same time, if we find a small set $F$ with $\phi(F)>0$ then automatically $\psi(F)>0$.

Theorem 6 summarizes results from $[4,5,13,14]$. Only [4] deals directly with the continuous time case.

For $\delta>0$, define $\tau_{F}(\delta)=\inf \{t \geq \delta \mid x(t) \in F\}$. In the continuous time case, $\tau_{F}(\delta)$ replaces the first time of return to the set $F$ from the discrete time setting.

Theorem 6. Assume a Markov process is non-explosive, $\psi$-irreducible and aperiodic with an attractive Doeblin set $F$. If there exists $\delta>0, \eta>0$ such that $V(x)=E_{x}\left[\exp \left(\eta \tau_{F}(\delta)\right)\right]$ is finite for all $x \in \mathcal{S}$ and $V(x)$ is uniformly bounded on $F$, then there exist a unique invariant probability measure $\mu(d x)$, constants $D>0$ and $\rho \in(0,1)$ such that for all $t \geq 0$ and $x \in \mathcal{S}$

$$
\left\|P_{x}(x(t) \in \cdot)-\mu(\cdot)\right\| \leq D V(x) \rho^{t},
$$

where $\|\cdot\|$ denotes the total variation norm of a measure.

Remark. The existence of an attractive Doeblin set $F$ implies that $F$ is a petite set for the resolvent chain with transition probabilities $U_{\lambda}(x, d y)=\int_{0}^{\infty} \lambda e^{-\lambda t} P_{x}(x(t) \in d y), \lambda>0$. Then $U_{\lambda}$ is Harris recurrent, implying that there exists an invariant measure $\mu(d x)$, not necessarily finite, for both the recurrent chain and $\{x(t)\}_{t \geq 0}$. Positive recurrence, defined as $E_{x}[\tau(\delta)]<\infty$ for all $x \in \mathcal{S}$ is necessary and sufficient to show that $\mu(d x)$ is a probability measure. The condition $V(x)<\infty$ is much stronger, and implies exponential ergodicity. 
Proof. The theorem is an immediate consequence of Theorems 6.2, 5.2 and 5.3 in [4] for the special function $f(x) \equiv 1$.

\section{REFERENCES}

[1] Baccelli, F.; Kim, K-B.; McDonald, D. R. Equilibria of a class of transport equations arising in congestion control. Queueing Syst. 55 (2007), no. 1, 18.

[2] Chiu, D.; Jain, R. Analysis of the increase/decrease algorithms for congestions avoidance in computer networks. Journal of Computer Networks, 17 (1989), 1-14.

[3] Martin Corless, Robert Shorten An ergodic AIMD algorithm with application to high-speed networks. International Journal of Control, Vol. 85, Iss. 6, 2012

[4] Down, D.; Meyn, S. P.; Tweedie, R. L. Exponential and uniform ergodicity of Markov processes. Ann. Probab. 23 (1995), no. 4, 1671-1691.

[5] Duflo, M. Random iterative models. Applications of Mathematics (New York), 34. Springer-Verlag, Berlin, 1997.

[6] V. Dumas, G. Guillemin, P. Robert A Markovian analysis of additive-increase multiplicative-decrease algorithms Advances in Applied Probability, 34 (1) (2002), pp. 85111

[7] Eun, D. Y. On the limitation of fluid-based approach for internet congestion control. IEEE International Conference on Computer Communications and Networks (ICCCN), San Diego, CA, Oct. 2005

[8] Eun, D. Y. Fluid approximation of a Markov chain for TCP/AQM with many flows. Preprint.

[9] Grigorescu, I., Kang, M. (2008) Steady State and Scaling Limit for a Traffic Congestion Model. European Series in Applied and Industrial Mathematics ESAIM: PS 14 (2010) 271-285

[10] Grigorescu, I., Kang, M. (2010) Recurrence and ergodicity for a continuous AIMD model, Preprint.

[11] Guillemin, F.; Robert, P.; Zwart, B. AIMD algorithms and exponential functionals. Annals of Applied Probability (2004).

[12] Kaspi, H.; Mandelbaum, A. On Harris Recurrence in Continuous Time. Mathematics of Operations Research, Vol. 19, No. 1 (1994), pp. 211-222

[13] Lacroix,J. Chaines de Markov et processus de Poisson, www.proba.jussieu.fr/cours/dea/markov.pdf

[14] Meyn, S. P.; Tweedie, R. L. Markov chains and stochastic stability. Communications and Control Engineering Series. Springer-Verlag London, Ltd., London, 1993.

[15] Pagano, M.; Secchi, R. A survey on TCP performance evaluation and modeling. Proc. of Int'l Working Conf. on Performance Modelling and Evaluation of Heterogeneous Networks (HET-NET 2004), July, Ilkley, UK

[16] Studl, S; Crisostomi, E; Middleton, R and Shorten, R. 2011. A Flexible Distributed Framework for Realising Electric and Plug-in Hybrid Vehicle Charging Policies. International Journal of Control, submitted. 
Department of Mathematics, University of Miami, Coral Gables, FL 33124-4250

E-mail address: igrigore@math.miami.edu

Department of Mathematics, North Carolina State University, Raleigh, NC 27695

E-mail address: kang@math.ncsu.edu 